\title{
Evaluation of Plazomicin, Tigecycline, and Meropenem Pharmacodynamic Exposure against Carbapenem-Resistant Enterobacteriaceae in Patients with Bloodstream Infection or Hospital-Acquired/ Ventilator-Associated Pneumonia from the CARE Study (ACHN-490-007)
}

Joseph L. Kuti · Aryun Kim • Daniel J. Cloutier · David P. Nicolau

Received: May 21, 2019 / Published online: June 28, 2019

(C) The Author(s) 2019

\section{ABSTRACT}

Introduction: CARE was a Phase 3, randomized study evaluating the efficacy and safety of plazomicin-based combination therapy compared with colistin-based combination therapy for the treatment of patients with bloodstream infections or hospital-acquired/ventilator-associated pneumonia due to carbapenem-resistant Enterobacteriaceae (CRE). Adjunctive therapies included either tigecycline or meropenem. We sought to understand the contribution of tigecycline and meropenem to plazomicin-treatedpatient outcomes by determining their observed pharmacodynamic exposures against baseline pathogens.

Enhanced digital features To view enhanced digital features for this article go to https://doi.org/10.6084/ m9.figshare.8299490.

Electronic supplementary material The online version of this article (https://doi.org/10.1007/s40121019-0251-4) contains supplementary material, which is available to authorized users.

J. L. Kuti · D. P. Nicolau ( $\square)$

Center for Anti-Infective Research and

Development, Hartford Hospital, 80 Seymour Street, Hartford, CT 06102, USA

e-mail: david.nicolau@hhchealth.org

A. Kim · D. J. Cloutier

Achaogen Inc., 1 Tower Place, Suite 300, South San

Francisco 94080, CA, USA
Methods: Blood samples collected for plazomicin therapeutic monitoring were assayed for tigecycline and meropenem concentrations. Population pharmacokinetic models were constructed for each antibiotic. Using the individual Bayesian posterior or a covariate-based model, concentration time profiles were simulated to estimate the pharmacodynamic exposures for each patient. Pharmacodynamic thresholds for plazomicin, tigecycline, and meropenem were a total area under the curve to minimum inhibitory concentration ratio (AUC/ $\mathrm{MIC}) \geq 85$, free $(f) \mathrm{AUC} / \mathrm{MIC} \geq 0.9$, and free time above the MIC ( $f \mathrm{~T}>\mathrm{MIC})$ of $\geq 40 \%$, respectively.

Results: Fifteen plazomicin-treated patients were included (12 received tigecycline, 4 received meropenem, 1 received both). Microbiological response was observed in 13 (86.7\%) and clinical efficacy was achieved in 11 (73.3\%). Plazomicin achieved its pharmacodynamic target in all 15 patients. Meropenem $f \mathrm{~T}>\mathrm{MIC}$ was $0 \%$ in all 4 patients, and tigecycline $f$ AUC/MIC was $\geq 0.9$ in $9(75 \%)$ patients. Overall, $6(40 \%)$ of 15 patients had a tigecycline or meropenem exposure below the requisite thresholds. Microbiological response and clinical efficacy were observed in $100 \%(6 / 6)$ and $83.3 \%(5 / 6)$ of patients with low threshold attainment by tigecycline and meropenem dosing regimens, respectively.

Conclusions: Plazomicin successfully achieved its requisite pharmacodynamic exposure, and 
these data suggest that optimization of tigecycline and meropenem therapy was not required for the combination to achieve microbiological response and clinical efficacy against serious CRE infections.

Trial Registration: ClinicalTrials.gov number, NCT01970371.

Funding: Achaogen, Inc.

Keywords: Aminoglycoside; Carbapenem; Glycylcycline; Pharmacodynamics; Pharmacokinetics

\section{INTRODUCTION}

Dissemination of carbapenem resistance has been observed in Klebsiella pneumoniae and Escherichia coli, as well as other Enterobacteriaceae [1, 2]. Among carbapenem-resistant Enterbacteriaceae (CRE), $\beta$-lactam resistance is chiefly due to the $K$. pneumoniae carbapenemase (KPC) enzyme, which has spread across the globe since the first isolation of the $b l a_{K P C}$ gene from a patient in 2001 [1]. Notably, pathogens carrying these enzymes are frequently resistant to other antimicrobial classes, including aminoglycosides and fluoroquinolones [3]. Aminoglycoside resistance in CRE can be due to a combination of impaired membrane permeability, efflux pumps, ribosomal alterations (e.g., 16s rRNA methyltransferase), or expression of aminoglycoside-modifying enzymes (AMEs), the latter of which are the most important cause of aminoglycoside resistance in Enterobacteriaceae [4].

Plazomicin is a next generation, semi-synthetic aminoglycoside that was recently approved in the US for the treatment of complicated urinary tract infections and pyelonephritis; it has also been studied for the treatment of bloodstream infections (BSI) caused by CRE [5]. The addition of side-chain substituents at targeted areas prevents most AMEs from inactivating plazomicin [5-7]. Study ACHN-490-007 (Combating Antibiotic-Resistant Enterobacteriaceae or CARE) was a Phase 3, randomized, open-label study evaluating the efficacy and safety of plazomicin compared with colistin for the treatment of patients with BSI and hospital-acquired/ventilator-associated bacterial pneumonia (HABP/VABP) due to CRE. Combination therapy with either tigecycline or meropenem, at the investigator's discretion, was required in both study arms. The primary end point was a composite of death from any cause at 28 days or clinically significant diseaserelated complications (SDRC). Patients randomized to combination therapy with plazomicin experienced an absolute reduction in the primary endpoint of $26 \%$ (95\% confidence interval - 55 to 6), and fewer deaths were observed at days 14-60 compared with the colistin-based regimen [8].

The achievement of optimal pharmacodynamic exposure is primarily responsible for an antibiotic's effectiveness in seriously ill patients with infection [9]. During the CARE Study, plazomicin was empirically administered at $15 \mathrm{mg} / \mathrm{kg}$ once daily or at an adjusted dosing regimen based on estimated creatinine clearance (CrCL) or type of renal replacement therapy. Subsequent dosing was determined based on therapeutic drug monitoring (TDM) to ensure plazomicin exposures were achieved within a pre-specified total drug area under the curve $\left(\right.$ AUC $\left._{0-24 \mathrm{~h}}\right)$ range of $210-315 \mathrm{mg} \mathrm{h} / \mathrm{L}$.

To better understand the effects of plazomicin in the context of using combination therapy, the pharmacodynamic exposure attainment of tigecycline and meropenem are also needed. We used remnant plasma collected for plazomicin TDM to determine concentrations of tigecycline or meropenem for pharmacokinetic and pharmacodynamic analyses. The purpose of the this study was to determine patient response to plazomicin treatment in the CARE study, while taking pharmacodynamic exposure attainment of tigecycline or meropenem into consideration.

\section{METHODS}

\section{Study Design}

This was a retrospective analysis of patient data collected during the ACHN-490-007 Study, here referred to as the CARE Study (ClinicalTrials.gov number, NCT01970371) [8]. This post hoc 
analysis was reviewed and approved by the Hartford Hospital Institutional Review Board. Informed consent was waived because all patient data were available and collected for the purposes of the CARE Study, and patient participants had already signed informed consent for data use.

\section{Patients and Antibiotic Dosing}

These analyses included 18 patients enrolled in Cohort 1 of the CARE Study who were randomized to receive plazomicin in combination with either tigecycline or meropenem. Patients were excluded if they did not meet study protocol criteria for the primary efficacy analysis population or if they did not receive at least 2 days of plazomicin therapy. During the trial, meropenem dosing regimens were recommended to be $2000 \mathrm{mg} \mathrm{q} 8 \mathrm{~h}$ with each dose administered as a 3-h infusion. Local protocols could be used to dose adjust for renal insufficiency. Higher tigecycline doses were also suggested, i.e., $200 \mathrm{mg}$ loading dose, followed by $100 \mathrm{mg} \mathrm{q} 12 \mathrm{~h}$; however, the standard dose (50 $\mathrm{mg} \mathrm{q} 12 \mathrm{~h}$ ) was permissible if it was the standard of care locally.

\section{Tigecycline and Meropenem Concentrations}

Cohort 1 patients under the original protocol had blood samples collected in $\mathrm{K}_{2}$ EDTA for determination of plazomicin concentration on day 1 and end of therapy at 6 time points, day 7 at 2 time points, and possibly on unscheduled TDM days at 2 time points following the plazomicin dose. After a protocol amendment, patients had blood samples collected for determination of plazomicin concentration on days 1 and 4 at 3 time points, day 8 at 2 time points, and possibly on unscheduled TDM days at 2 time points following the plazomicin dose. These samples were stored at $-80^{\circ} \mathrm{C}$ after determination of plazomicin concentrations. Remnant plasma from these samples was thawed and tested for tigecycline or meropenem concentration by Alturas Analytics (Moscow, ID, USA) using validated high- performance liquid chromatography with tandem mass spectrometry assay. Details of the assay can be found in the Supplementary Data. The mean \% coefficient of variation $(\mathrm{CV})$ and \% bias across all quality control levels for tigecycline were within $9.9 \%$ and $6.4 \%$, respectively. The mean \% CV and \% bias for quality controls for meropenem were within $7.6 \%$ and $10.7 \%$, respectively.

\section{Data Collection and Definitions}

Additional data collected included: time of sample collection, start of infusion and end of infusion times of all tigecycline or meropenem doses administered prior to the collected samples, doses received, list of prior and concomitant medications, plazomicin $\mathrm{AUC}_{0-24 \mathrm{~h}}$ on day 1 of therapy [10], the plazomicin, tigecycline or meropenem MIC of the baseline CRE pathogen, age, gender, height, weight, daily serum creatinine, daily creatinine clearance, source of infection, all-cause mortality and presence of SDRC at day 28, and microbiological response. The primary efficacy endpoint was defined as clinical cure if the patient was alive at day 28 and had no SDRC as defined by the protocol. Failure was defined as death at day 28, presence of a SDRC, or both. Microbiological response was defined at test of cure ( $7 \pm 2$ days after end of therapy); eradication or persistence was defined as absence or presence, respectively, of the CRE on follow-up cultures at test of cure. Presumed eradication or presumed persistence was defined based on clinical response in the absence of culture data.

\section{Pharmacokinetics}

Population pharmacokinetic models were developed for tigecycline and meropenem using the non-parametric adaptive grid (NPAG) program in Pmetrics for R (Laboratory of Applied Pharmacokinetics and Bioinformatics, Los Angeles, CA, USA) [11]. Visual inspection of data and Akaike information criterion were used to differentiate multiple models. Once converged, the NPAG Bayesian posterior 
pharmacokinetic parameter estimates for each patient from the CARE Study were extracted.

\section{Tigecycline and Meropenem Exposures}

Individual total drug tigecycline and meropenem concentration profiles were simulated at 12-min intervals over the first $96 \mathrm{~h}$ of therapy for each patient based on their Bayesian posterior parameter estimates and the dosages received during the clinical trial. Tigecycline total drug concentrations were corrected for protein binding using a fixed ratio of 0.20 for the fraction unbound, as previously described [12]. Meropenem concentrations were corrected for protein binding by applying a fixed ratio of 0.98 to all resulting concentration values $[13,14]$. For the few patients with no concentration data available, covariate-based population models from Rubino et al. (tigecycline) [15] and Crandon and colleagues (meropenem) [13] were employed to estimate pharmacokinetic parameters.

\section{Pharmacodynamic Analyses}

For each individual patient receiving tigecycline, the a priori primary pharmacodynamic endpoint was the frequency of patients who achieved a fAUC/MIC at steady-state of tigecycline therapy $\geq 0.9$, which was associated with successful clinical response in patients with hospital-acquired pneumonia [12]. Steady-state fAUC exposure was calculated by the fraction unbound multiplied by the 24-h maintenance dose, divided by total body clearance for the patient. The MIC of the CRE causative pathogen was used to define exposure. For patients receiving meropenem, the $f \mathrm{~T}>\mathrm{MIC}$ was calculated over a 24-h period for the first day of meropenem therapy. The a priori primary pharmacodynamic endpoint was the frequency of patients who achieved at least $40 \% f \mathrm{~T}>\mathrm{MIC}$ on day 1 , which was the exposure required for approximately a $2 \log$ reduction in colony forming units in the neutropenic mouse thigh infection model against Escherichia coli and Pseudomonas aeruginosa [14]. Day 1 plazomicin total drug 24-h AUC/MIC exposure for each patient was calculated from the provided $\mathrm{AUC}_{0-24 \mathrm{~h}}$. The percent of patients who achieved a total drug 24-h AUC/MIC $\geq 85$ was assessed for plazomicin; this exposure was associated with $1-\log _{10}$ reduction in the neutropenic murine thigh infection model against Enterobacteriaceae [16].

\section{Statistical Analyses}

Descriptive statistics were used to characterize the percent of patients receiving meropenem or tigecycline who achieved the pharmacodynamic threshold and association with microbiological response and the primary efficacy endpoint. Fisher's exact test (SigmaPlot v.13; Systat, San Jose, CA, USA) was used to compare the proportion of patients who achieved their tigecycline or meropenem exposure thresholds with microbiological response and efficacy endpoints. Attainment of the plazomicin pharmacodynamic threshold was examined in relation to the meropenem/tigecycline exposures achieved.

\section{RESULTS}

\section{Patients}

Of the 18 plazomicin-treated patients enrolled in Cohort 1 of the CARE Study, 15 met inclusion/exclusion criteria for this analysis, 1 patient was excluded due to absence of CRE at baseline, and 2 patients were excluded after only receiving a single dose of plazomicin due to documented plazomicin resistance in the baseline CRE. From these 15 patients, 12 were treated with tigecycline and 4 were treated with meropenem; 1 patient received meropenem for study days $1-4$, followed by tigecycline for days $4-14$. Three of the 15 patients were excluded from pharmacokinetic model development because original signed consent forms did not permit analysis of remnant plasma samples for tigecycline or meropenem concentrations. Therefore, of the 12 tigecycline- and 4 meropenem-treated patients, 11 and 2, respectively, contributed concentration data. The single 
Table 1 Included patient characteristics and availability of tigecycline or meropenem concentration data from plazomicin treated patients in cohort 1 of the CARE Study

\begin{tabular}{|c|c|c|c|c|c|c|c|c|}
\hline $\begin{array}{l}\text { Patient } \\
\text { number }\end{array}$ & $\begin{array}{l}\text { APACHE } \\
\text { II }\end{array}$ & $\begin{array}{l}\text { Infection type } \\
\text { (source) }\end{array}$ & $\begin{array}{l}\text { Adjunctive } \\
\text { treatment }\end{array}$ & $\begin{array}{l}\text { PK } \\
\text { patient }^{a}\end{array}$ & $\begin{array}{l}\text { Treatment } \\
\text { duration }\end{array}$ & $\begin{array}{l}\text { Baseline CrCL } \\
(\mathrm{mL} / \mathrm{min})\end{array}$ & $\begin{array}{l}\mathrm{ABW} \\
(\mathrm{kg})\end{array}$ & $\begin{array}{l}\text { Height } \\
(\mathrm{cm})\end{array}$ \\
\hline 1 & 15 & BSI (primary) & Meropenem & No & 14 & 72 & 70 & 182 \\
\hline 2 & 18 & BSI (primary) & Meropenem & No & 14 & 62 & 80 & 170 \\
\hline 3 & 25 & BSI (primary) & Tigecycline & Yes & 11 & $18^{\mathrm{c}}$ & 75 & 148 \\
\hline $4^{b}$ & 23 & VAP & $\begin{array}{l}\text { Meropenem, } \\
\text { tigecycline }\end{array}$ & Yes & 14 & 150 & 165 & 168 \\
\hline 5 & 20 & VAP & Tigecycline & Yes & 3 & 35 & 90 & 167 \\
\hline 6 & 20 & BSI (primary) & Tigecycline & Yes & 12 & 74 & 135 & 178 \\
\hline 7 & 16 & BSI (primary) & Tigecycline & Yes & 15 & 196 & 95 & 185 \\
\hline 8 & 17 & BSI (primary) & Tigecycline & Yes & 12 & 176 & 65 & 168 \\
\hline 9 & 23 & BSI (primary) & Tigecycline & Yes & 10 & $39^{c}$ & 75 & 165 \\
\hline 10 & 19 & BSI (primary) & Tigecycline & Yes & 14 & $19^{c}$ & 70 & 182 \\
\hline 11 & 19 & BSI (catheter) & Tigecycline & Yes & 12 & 153 & 100 & 175 \\
\hline 12 & 19 & BSI (primary) & Tigecycline & Yes & 14 & 87 & 75 & 161 \\
\hline 15 & 24 & $\begin{array}{l}\text { BSI (intra- } \\
\text { abdominal) }\end{array}$ & Meropenem & Yes & 13 & 16 & 60 & 174 \\
\hline 16 & 23 & VAP & Tigecycline & Yes & 2 & $30^{c}$ & 90 & 172 \\
\hline 17 & 31 & $\begin{array}{l}\text { BSI (intra- } \\
\text { abdominal) }\end{array}$ & Tigecycline & No & 12 & 30 & 86 & 154 \\
\hline
\end{tabular}

$B S I$ bloodstream infection, $V A P$ ventilator-associated pneumonia, $P K$ pharmacokinetics (concentrations available for model construction), $\operatorname{Cr} C L$ creatinine clearance, $A B W$ actual body weight at baseline

${ }^{a}$ PK patients included 12 patients who provided concentration data for either tigecycline, meropenem, or both. These patients were used to construct population pharmacokinetic models. The remaining 3 patients were included in the pharmacodynamic analyses only, as their exposure was estimated from covariate-based demographic models

b Patient \#4 received meropenem for study days 1-4, followed by tigecycline for study days 4-14

${ }^{c}$ Four tigecycline patients received renal replacement therapy during the study

patient who received meropenem and tigecycline contributed concentration data for both drugs. The remaining patients had tigecycline or meropenem exposure estimated via covariate-derived models. A list of the 15 patients included is provided in Table 1 . Patients were $67 \pm 13$ years of age (range 33-84 years), and 11 (73\%) were male. Causative pathogens, source, and antibiotic MICs are listed in Table 2. All CRE were K. pneumoniae except for a single Enterobacter aerogenes. Median (range) plazomicin, tigecycline, and meropenem minimum inhibitory concentration (MIC) for the isolates were $0.25 \mathrm{mg} / \mathrm{L}(0.12-0.5), 1 \mathrm{mg} / \mathrm{L}(0.5-8)$, and $64 \mathrm{mg} / \mathrm{L}$ (4-256), respectively.

\section{Plazomicin, Tigecycline, and Meropenem Pharmacokinetics}

The 12 patients with available concentration data provided between 2 and 16 samples each, frequently spread over multiple doses, thus resulting in 89 samples from 11 patients available for tigecycline and 14 samples from 2 
Table 2 List of baseline CRE by patient, source, and plazomicin, meropenem, and tigecycline MIC

\begin{tabular}{llllll}
\hline $\begin{array}{l}\text { Patient } \\
\text { number }\end{array}$ & Baseline pathogen & Source of culture & $\begin{array}{l}\text { Plazomicin } \\
\text { MIC }(\mathbf{m g} / \mathbf{L})\end{array}$ & $\begin{array}{l}\text { Meropenem } \\
\text { MIC }(\mathbf{m g} / \mathbf{L})\end{array}$ & $\begin{array}{l}\text { Tigecycline } \\
\text { MIC }(\mathbf{m g} / \mathbf{L})\end{array}$ \\
\hline 1 & Klebsiella pneumoniae & Blood & 0.12 & 64 & 8 \\
2 & Klebsiella pneumoniae & Blood & 0.12 & 64 & 4 \\
3 & Klebsiella pneumoniae & Blood & 0.25 & 64 & 0.5 \\
4 & Klebsiella pneumoniae & Endotracheal aspirate & 0.5 & 128 & 0.5 \\
5 & Klebsiella pneumoniae & Endotracheal aspirate & 0.5 & 64 & 2 \\
6 & Klebsiella pneumoniae & Blood & 0.5 & 128 & 1 \\
7 & Klebsiella pneumoniae & Blood & 0.5 & 128 & 1 \\
8 & Klebsiella pneumoniae & Blood & 0.25 & 64 & 0.5 \\
9 & Klebsiella pneumoniae & Blood & 0.12 & 4 & 0.5 \\
10 & Klebsiella pneumoniae & Blood & 0.5 & 4 & 1 \\
11 & Enterobacter aerogenes & Blood & 0.25 & 4 & 1 \\
12 & Klebsiella pneumoniae & Blood & 0.25 & 16 & 1 \\
15 & Klebsiella pneumoniae & Blood & 0.25 & 256 & 1 \\
16 & Klebsiella pneumoniae & Protected-specimen brush & 0.25 & 64 & 0.5 \\
17 & Klebsiella pneumoniae & Blood & 0.12 & 4 & 2 \\
\hline
\end{tabular}

CRE carbapenem-resistant Enterobactericeae, $M I C$ minimum inhibitory concentration

patients available for meropenem pharmacokinetic analyses. Raw concentration data are provided in Table S1 of the supplementary data. Remnant concentration data initially for the purpose of plazomicin TDM was sufficient to determine tigecycline and meropenem concentrations. Plazomicin mean \pm standard deviation (range) for total drug $\mathrm{AUC}_{0-24 \mathrm{~h}}$ on day 1 of therapy was $263.4 \pm 98.7 \mathrm{mg} \mathrm{h} / \mathrm{L}(139.0-444.9)$ for the 15 included patients.

Tigecycline concentration data for the 11 patients fitted a two-compartment model. The observed versus population and individual predicted concentration plots are displayed in Fig. 1. Final Bayesian posterior individual estimates for pharmacokinetic parameters are provided in Table 3 . The mean \pm standard deviation values for clearance and volume of the central compartment were $24.8 \pm 7.7 \mathrm{~L} / \mathrm{h}$ and $84.9 \pm 41.5 \mathrm{~L}$, respectively. The single tigecycline patient not contributing concentration data had an $\mathrm{AUC}_{0-24 \mathrm{~h}}$ calculated from total body clearance, which was estimated from the covariate-based pharmacokinetic model [14]. Mean \pm standard deviation (range) tigecycline fAUC over $24 \mathrm{~h}$ at steady-state for all 12 patients was $1.27 \pm 0.55 \mathrm{mg} \mathrm{h} / \mathrm{L}(0.54-2.16)$. A comparison of model-derived pharmacokinetic parameter estimates and AUC exposure versus covariate-based estimates is provided in Tables S2 and S3.

Meropenem concentration data for the two patients fitted a two-compartment model. The observed versus individual predicted concentration plots for these two patients are displayed in Fig. 2a, b. While concentration data for patient 4 (Fig. 2a) achieved a good fit to the observed data, this was not the case with patient 15 (Fig. 2b), whose predicted concentrations were significantly lower than observed. Therefore, covariate-based equations were used to estimate meropenem pharmacokinetics for patient 15 and the two remaining meropenem patients ( 1 and 2 ) who had no concentration 

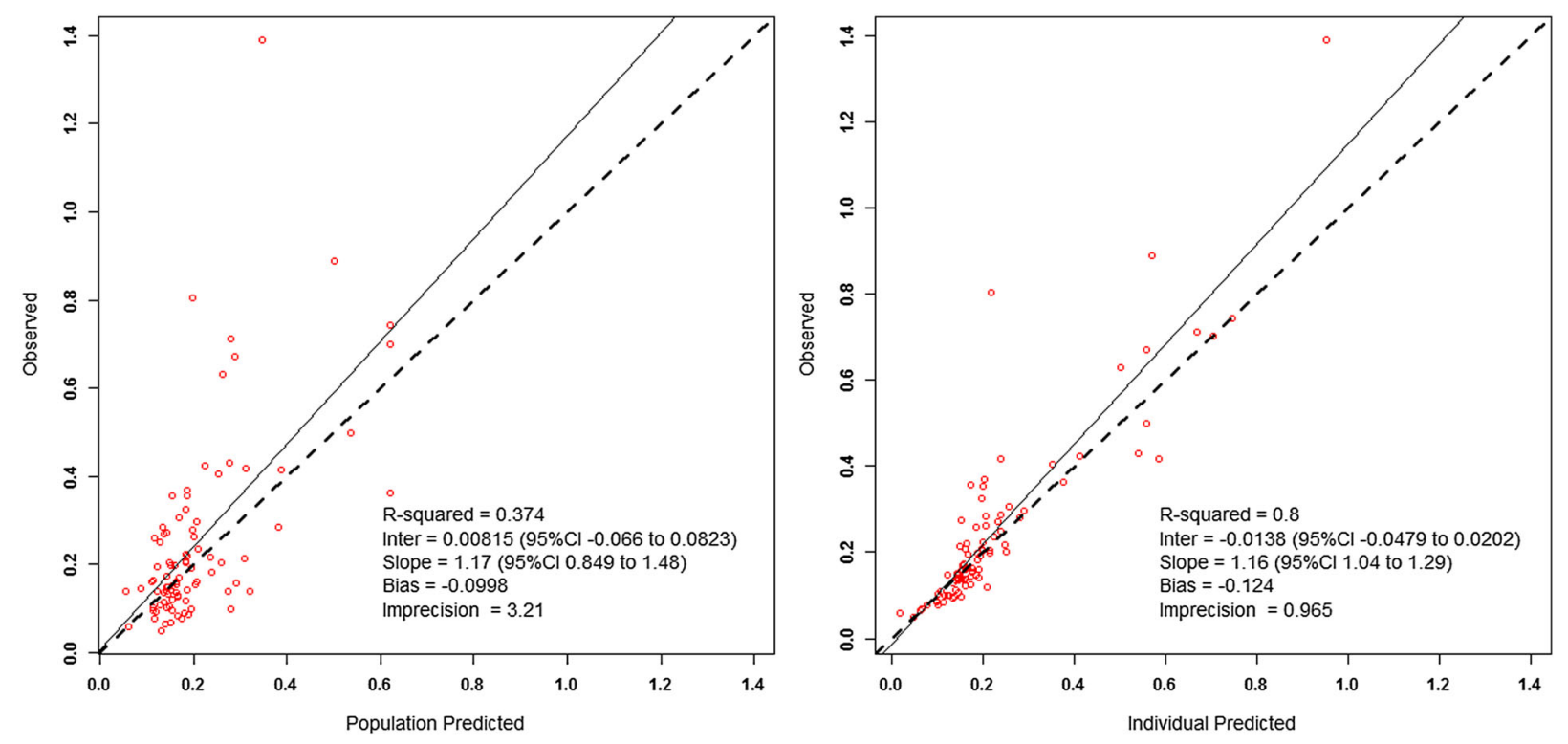

Fig. 1 Observed versus (left) population predicted and (right) individual predicted tigecycline concentrations from the final model

data available (Table 3). A comparison of modelderived pharmacokinetic parameter estimates versus covariate-based estimates is provided in Table S4.

\section{Pharmacodynamic Analyses}

The mean \pm standard deviation (range) for plazomicin total drug AUC/MIC on day 1 was $1297 \pm 1132$ (278-3708). All 15 patients (100\%) achieved the target plazomicin pharmacodynamic threshold (Table 4). Steady-state tigecycline fAUC/MIC and day 1 meropenem-free time above the MIC ( $f$ T $>$ MIC) are listed in Table 4. Nine of 12 (75\%) tigecycline treated patients achieved a fAUC/MIC of at least 0.9. Due to high MICs against the baseline CRE, none of the 4 meropenem patients achieved at least $40 \% f \mathrm{~T}>\mathrm{MIC}$, and none had any concentrations above $64 \mathrm{mg} / \mathrm{L}$ during the first $24 \mathrm{~h}$ of therapy. The single patient who received meropenem followed by tigecycline did not attain meropenem exposure thresholds, but did obtain the target for tigecycline; this patient was counted as achieving the pharmacodynamic exposure target. Therefore, 6 of 15 (40\%) patients in the CARE Study did not achieve the requisite pharmacodynamic threshold for the adjunctive antibiotic.

A positive microbiological response was observed in 13 of the $15(86.7 \%)$ patients, and the primary clinical efficacy endpoint was cure in 11 of the $15(73.3 \%)$ patients. There was no significant relationship between tigecycline or meropenem achievement of pharmacodynamic thresholds and microbiological response or clinical response for the 15 patients (Table 5). Overall, the microbiological and clinical response rates did not differ based on whether the pharmacodynamic target was achieved for the adjunctive agent. For the 6 patients who achieved the pharmacodynamic threshold for only plazomicin and not for the adjunctive agent, the positive microbiological response and clinical response rates were $6 / 6(100 \%)$ and 5/6 (83.3\%), respectively.

\section{DISCUSSION}

Due to fear of adaptive resistance development, aminoglycosides have historically been administered in combination with other antibiotics for serious Gram-negative infections [17-19]. This unstable resistance is predominantly 
Table 3 Median individual Bayesian posterior parameter estimates for the 11 tigecycline and 1 meropenem patient plus covariate-based parameter estimates for 1 tigecycline and 3 meropenem patients used to estimate pharmacodynamic exposure

\begin{tabular}{|c|c|c|c|c|}
\hline \multirow[t]{2}{*}{ Antibiotic/patient number } & \multicolumn{4}{|c|}{ Pharmacokinetic parameter estimates $^{\mathrm{a}}$} \\
\hline & $\overline{\mathbf{C L}(\mathbf{L} / \mathrm{h})}$ & Vc (L) & $\operatorname{kcp}\left(h^{-1}\right)$ & kpc $\left(h^{-1}\right)$ \\
\hline \multicolumn{5}{|l|}{ Tigecycline } \\
\hline 3 & 22.3 & 118.9 & 5.688 & 1.883 \\
\hline 4 & 37.0 & 100.2 & 1.046 & 0.375 \\
\hline 5 & 19.0 & 89.1 & 1.945 & 1.269 \\
\hline 6 & 15.0 & 79.3 & 2.575 & 0.299 \\
\hline 7 & 37.0 & 100.3 & 1.045 & 0.376 \\
\hline 8 & 37.0 & 100.3 & 1.045 & 0.375 \\
\hline 9 & 22.5 & 149.0 & 3.583 & 1.986 \\
\hline 10 & 19.5 & 89.1 & 4.152 & 1.268 \\
\hline 11 & 24.2 & 26.1 & 7.885 & 0.110 \\
\hline 12 & 19.4 & 21.9 & 4.195 & 0.490 \\
\hline 16 & 19.2 & 88.6 & 3.540 & 1.262 \\
\hline $17^{\mathrm{b}}$ & 17.1 & $\mathrm{~N} / \mathrm{A}$ & $\mathrm{N} / \mathrm{A}$ & $\mathrm{N} / \mathrm{A}$ \\
\hline \multicolumn{5}{|l|}{ Meropenem } \\
\hline $1^{\mathrm{c}}$ & 10.2 & 16.7 & 0.487 & 0.647 \\
\hline $2^{c}$ & 9.5 & 16.5 & 0.487 & 0.647 \\
\hline 4 & 22.0 & 40.9 & 9.95 & 4.41 \\
\hline $15^{\mathrm{c}}$ & 6.3 & 14.3 & 0.487 & 0.647 \\
\hline
\end{tabular}

$C L$ clearance, $V c$ volume of distribution of the central compartment, $k c p$ and $k p c$ intercompartment transfer constants between central and peripheral compartments and vice versa, respectively, $f A U C / M I C$ free area under the curve to minimum inhibitory concentration ratio, $f T>M I C$ free time above the minimum inhibitory concentration, $N / A$ not available

a All pharmacokinetic parameter estimates are derived from the Bayesian posterior model fits in Pmetrics, except for patients 1, 2, 15, and 17 (see footnotes)

${ }^{b}$ Concentration data not available for this patient. Exposure estimated using a covariate-based model [15], as 24-h maintenance dose divided by $\mathrm{CL}$, where $\mathrm{CL}=[(9.6+(10.2 \times \mathrm{BSA}-1.73))]+[(0.0638 \times(\mathrm{CrCL}-100)]$, where BSA is body surface area and CrCL is baseline creatinine clearance

${ }^{c}$ Concentration data not available for two patients ( 1 and 2$)$, and observed versus predicted concentrations were poorly fit for patient 15. Exposure estimated using a covariate-based model [13], based on clearance [CL $=(0.392+0.003 \times$ $\mathrm{CrCL})]$, volume of the central compartment $[\mathrm{Vc}=0.239 \times$ adjusted body weight, where adjusted body weight $=$ $\mathrm{IBW}+[0.4 \times(\mathrm{ABW}-\mathrm{IBW})$ or if adjusted body weight is $<20 \%$ above ideal body weight (IBW), then actual body weight used $(\mathrm{ABW})]$, and median population estimates for $\mathrm{kcp}$ and $\mathrm{kpc}$

described in Pseudomonas aeruginosa and can be largely avoided by dose optimization with highdose, extended-interval regimens. Nonetheless, as an aminoglycoside antibiotic, plazomicin was studied in combination with either tigecycline or meropenem against CRE BSI and HABP/ VABP in the CARE Study. Microbiological and clinical outcomes in the patients randomized to 


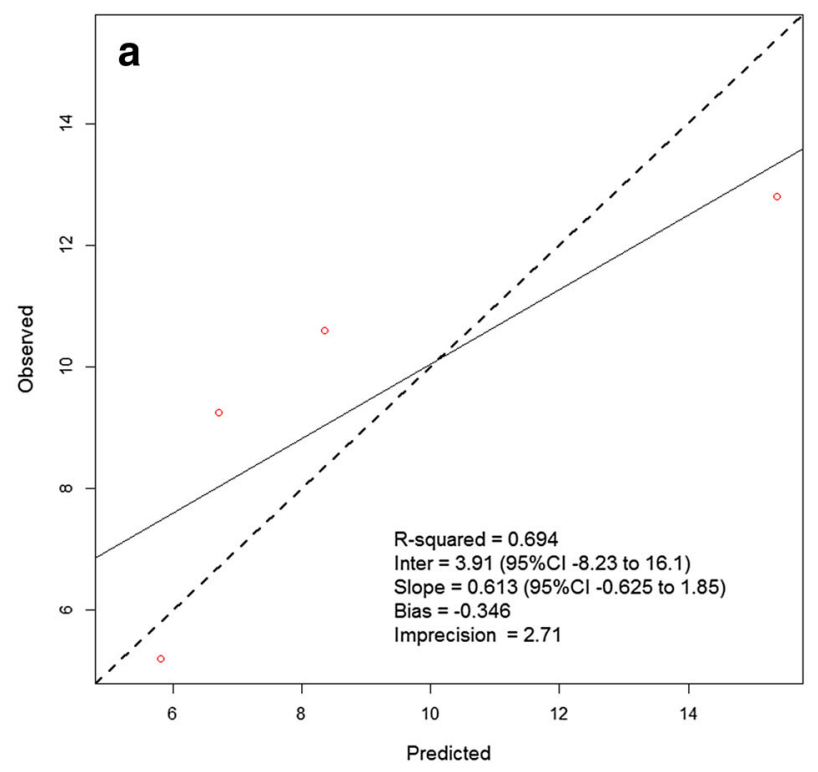

Fig. 2 Observed versus Bayesian posterior individual predicted concentrations for $\mathbf{a}$ patient 4 and $\mathbf{b}$ patient 15 , who received meropenem in the CARE Study. While

receive plazomicin were favorable, with mortality reductions observed over patients receiving colistin [8]. The contribution of tigecycline or meropenem to these outcomes has not been established. Data from a recent murine sepsis infection model found that monotherapy with plazomicin at human-simulated concentrations resulted in survival similar to a combination that included plazomicin plus tigecycline or meropenem against CRE [20]. This study sought to evaluate the pharmacodynamic exposure attained individually by patients receiving tigecycline or meropenem in the plazomicin CARE study.

Plazomicin dosing regimens in the trial achieved its pharmacodynamic exposure (i.e., total drug AUC/MIC $\geq 85$ ) in all critically ill patients in the study, regardless of hemodynamic stability or baseline renal function. This pharmacodynamic threshold was significantly associated with a $1-\log _{10}$ reduction in neutropenic murine thigh infection studies against Enterobacteriaceae [16]. Day $1 \mathrm{AUC}_{0-24 \mathrm{~h}}$ was $263.4 \pm 98.7 \mathrm{mg} \mathrm{h} / \mathrm{L}$ with an observed range of $139.0-444.9 \mathrm{mg} \mathrm{h} / \mathrm{L}$. TDM was conducted to maintain $\mathrm{AUC}_{0-24 \mathrm{~h}}$ exposure between 210 and $315 \mathrm{mg} \mathrm{h} / \mathrm{L}$. Four patients obtained initial

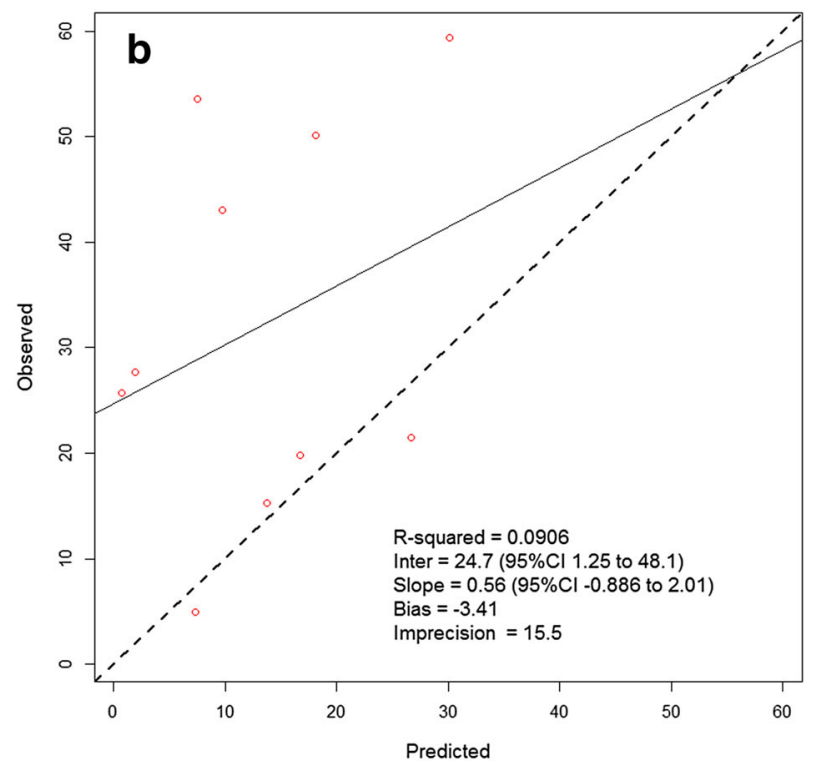

precision was reasonable for patient 4 , individual Bayesian parameter estimates for patient 15 resulted in predicted concentrations significantly different from observed

AUC $_{0-24 \mathrm{~h}}$ exposure below $210 \mathrm{mg} \mathrm{h} / \mathrm{L}$, but, due to low plazomicin MICs of these CRE, these patients successfully achieved the pharmacodynamic threshold. All but three patients would have achieved the requisite pharmacodynamic threshold on day 1 with MICs up to $2 \mathrm{mg} / \mathrm{L}$. These observations are supported by Monte Carlo simulation data demonstrating high probabilities of target attainment for the approved dosing regimen at MICs up to $2 \mathrm{mg} / \mathrm{L}$ [16].

Tigecycline was more frequently administered in combination with plazomicin compared with meropenem in the CARE Study. Twelve patients received tigecycline, of which 11 provided concentration data for the population pharmacokinetic model. Tigecycline pharmacokinetics have been previously described in critically ill patients $[15,21,22]$. We observed tigecycline clearance to be $24.8 \mathrm{~L} / \mathrm{h}$ with $\sim 30 \%$ variation among patients, estimates which were similar to Rubino et al.(19.2 $\mathrm{L} / \mathrm{h}, 40.4 \% \mathrm{CV})$ and Borsuk-De Moor et al. (22.1 $\mathrm{L} / \mathrm{h}, 17.3 \% \mathrm{CV}$ ) $[15,22]$. Volume of the central compartment was large and varied between patients, as also noted in these other studies. Four patients receiving tigecycline were also 
Table 4 Plazomicin, tigecycline, and meropenem pharmacodynamic exposure compared with microbiological and clinical response

\begin{tabular}{|c|c|c|c|c|c|c|c|}
\hline Patient & $\begin{array}{l}\text { Plazomicin } \\
\text { AUC/MIC } \\
(\text { MIC })^{\mathrm{a}}\end{array}$ & $\begin{array}{l}\text { Adjunct } \\
\text { antibiotic }\end{array}$ & $\begin{array}{l}\text { Tigecycline } \\
\text { fAUC/MIC } \\
\text { (MIC) }\end{array}$ & $\begin{array}{l}\text { Meropenem } \\
f \Gamma>\text { MIC } \\
(\text { MIC })\end{array}$ & $\begin{array}{l}\text { Tigecycline/ } \\
\text { meropenem PD } \\
\text { threshold } \\
\text { achieved }^{\mathbf{b}}\end{array}$ & $\begin{array}{l}\text { Microbiological } \\
\text { response at } \\
\text { TOC }\end{array}$ & $\begin{array}{l}\text { Clinical } \\
\text { response } \\
\text { at } 28 \text { days }\end{array}$ \\
\hline 1 & $3333(0.12)$ & Meropenem & N/A & $0 \%(64)$ & No & Eradication & Cure \\
\hline 2 & $3708(0.12)$ & Meropenem & N/A & $0 \%(64)$ & No & Eradication & Cure \\
\hline 3 & $1048(0.25)$ & Tigecycline & $1.79(0.5)$ & $\mathrm{N} / \mathrm{A}$ & Yes & Eradication & Cure \\
\hline $4^{c}$ & $461(0.5)$ & $\begin{array}{l}\text { Tigecycline } \\
\text { and } \\
\text { meropenem }\end{array}$ & $1.08(0.5)$ & $0 \%(128)$ & Yes/no & $\begin{array}{l}\text { Presumed } \\
\text { eradication }\end{array}$ & Cure \\
\hline 5 & $476(0.5)$ & Tigecycline & $1.05(2)$ & N/A & Yes & $\begin{array}{l}\text { Presumed } \\
\text { persistence }\end{array}$ & Failure \\
\hline 6 & $836(0.5)$ & Tigecycline & $1.33(1)$ & N/A & Yes & Eradication & Cure \\
\hline 7 & $278(0.5)$ & Tigecycline & $0.54(1)$ & N/A & No & Eradication & Cure \\
\hline 8 & $733(0.25)$ & Tigecycline & $2.16(0.5)$ & N/A & Yes & Eradication & Failure \\
\hline 9 & $3003(0.12)$ & Tigecycline & $1.78(0.5)$ & N/A & Yes & Eradication & Cure \\
\hline 10 & $480(0.5)$ & Tigecycline & $1.03(1)$ & N/A & Yes & Eradication & Cure \\
\hline 11 & $1027(0.25)$ & Tigecycline & $0.83(1)$ & N/A & No & Eradication & Cure \\
\hline 12 & $1022(0.25)$ & Tigecycline & $1.03(1)$ & N/A & Yes & Eradication & Cure \\
\hline 15 & $622(0.25)$ & Meropenem & N/A & $0 \%(256)$ & No & Eradication & Failure \\
\hline 16 & $583(0.25)$ & Tigecycline & $2.08(0.5)$ & N/A & Yes & $\begin{array}{l}\text { Presumed } \\
\text { persistence }\end{array}$ & Failure \\
\hline 17 & $1844(0.12)$ & Tigecycline & $0.59(2)$ & $\mathrm{N} / \mathrm{A}$ & No & Eradication & Cure \\
\hline
\end{tabular}

$N / A$ not available, $M I C$ minimum inhibitory concentration, $A U C / M I C$ total drug area under the curve/MIC, $f A U C / M I C$ free area under the curve/MIC, $f T>M I C$ free time above the MIC as a percent of the dosing interval, $P D$ pharmacodynamics, TOC test of cure

a Plazomicin AUC/MIC $\geq 85$ on day 1 was defined as the pharmacodynamic target

b $f$ AUC/MIC $\geq 0.9$ at steady-state was defined as the pharmacodynamic target for tigecycline. For meropenem, a threshold $\geq 40 \% f \Gamma>$ MIC on day 1 was defined as the pharmacodynamic target

c Patient 4 received meropenem for study days $1-4$, followed by tigecycline for study days $4-14$; this patient is counted as exposure threshold achieved due to attainment of tigecycline PD target

receiving renal replacement therapy, but this therapy has not been found to widely affect tigecycline clearance $[22,23]$. It should also be noted that the single patient without available concentration data had a clearance of $17.1 \mathrm{~L} / \mathrm{h}$ when estimated by the covariate-based model [15], which was consistent with the other patients in the cohort (Tables 3, S3).
The pharmacodynamic threshold applied for tigecycline was a steady-state $f$ AUC/MIC $\geq 0.9$, which was associated with successful clinical response in patients with hospital-acquired pneumonia [12]. Although other pharmacodynamic thresholds have been described for tigecycline $[24,25]$, this threshold is the only one derived in critically ill patients that most closely 
Table 5 Summary of microbiological and clinical response versus achievement of tigecycline or meropenem pharmacodynamic threshold exposure

\begin{tabular}{|c|c|c|c|c|c|c|}
\hline \multirow[t]{2}{*}{ Antibiotic } & \multicolumn{3}{|c|}{ Microbiological response } & \multicolumn{3}{|c|}{ Clinical response } \\
\hline & Eradiction & Persistance & $\overline{p \text { value }}$ & Cure & Failure & $\begin{array}{l}P \text { - } \\
\text { value }\end{array}$ \\
\hline Tigecycline & $n=10$ & $n=2$ & & $n=9$ & $n=3$ & \\
\hline$f A U C / M I C \geq 0.9$ & $7(70.0)$ & $2(100)$ & $\mathrm{NC}$ & $6(66.6)$ & $3(100)$ & $\mathrm{NC}$ \\
\hline Meropenem & $n=4$ & $n=0$ & & $n=3$ & $n=1$ & \\
\hline$f \Gamma>M I C \geq 40 \%$ & 0 & 0 & $\mathrm{NC}$ & 0 & 0 & $\mathrm{NC}$ \\
\hline Both antibiotics ${ }^{\mathrm{a}}$ & $n=13$ & $n=2$ & & $n=11$ & $n=4$ & \\
\hline Achieved PD threshold & $7(53.8)$ & $2(100)$ & 0.486 & $6(54.5)$ & $3(75.0)$ & 0.604 \\
\hline
\end{tabular}

$f A U C / M I C$ free area under the curve/MIC, $f T>M I C$ free time above the MIC, $P D$ pharmacodynamic, $N C$ not calculated

${ }^{a}$ Patient 4 received meropenem for study days $1-4$, followed by tigecycline for study days $4-14$; this patient is counted as exposure threshold achieved due to attainment of tigecycline PD target

resembles the population enrolled in the CARE Study. Furthermore, this target was generated in patients who were in part receiving combination therapy during the original registration studies for tigecycline. We observed that 9 $(75 \%)$ of the patients receiving tigecycline achieved a fAUC/MIC $\geq 0.9$. Notably, the 3 patients who did not achieve this threshold were defined as microbiological responders and clinical cures, suggesting that plazomicin exposure or the combination of the two antibiotics had a substantial role in outcome. As a sensitivity analysis, we also applied the total drug AUC/MIC target $\geq 6.96$, which was derived from patients with intra-abdominal infections [24]; this pharmacodynamic exposure threshold was achieved in only 4 of 12 (33.3\%) (data not shown). Eradication or cure was observed in 7 of the 8 patients with suboptimal tigecycline exposure.

Only two meropenem patients provided concentration data in the CARE Study. Unfortunately, only predicted concentrations for one were adequately similar to observed to permit inclusion into the individual patient simulations. For the remaining three meropenem patients, pharmacokinetic parameters were estimated from a covariate-based model [13]. Notably, the fitted parameter estimates for patient 5 resembled the covariate-based estimates for that patient (Table S4). Nonetheless, the meropenem MICs of the baseline CRE in all patients who received adjunctive meropenem were $\geq 64 \mathrm{mg} / \mathrm{L}$. Even with protocol recommended dosing regimens of $2000 \mathrm{mg}$ $\mathrm{q} 8 \mathrm{~h}$ as $3 \mathrm{~h}$ prolonged infusions, none of these patients achieved any concentration above $64 \mathrm{mg} / \mathrm{L}$ at any point during their observed $(n=2)$ (Table S1) or simulated $(n=4)$ concentration time profiles. The pharmacodynamic threshold for meropenem is $40 \% f \mathrm{~T}>$ MIC [14]. Higher $f \mathrm{~T}>$ MIC thresholds have been observed in human studies $[26,27]$, but applying more aggressive meropenem targets would not have resulted in different observations. Among these four patients who were unable to achieve optimal meropenem $f \mathrm{~T}>\mathrm{MIC}$, all achieved a positive microbiological response, and 3 (75\%) achieved a positive clinical response.

Overall, $40 \%(6 / 15)$ of patients receiving plazomicin in combination with tigecycline or meropenem did not achieve optimal pharmacodynamic threshold for the adjunct agent, and only plazomicin was observed to achieve its target. Despite this, microbiological response and the clinical efficacy endpoint of cure were observed in $100 \%(6 / 6)$ and $83.3 \%(5 / 6)$, respectively. The one patient who received both meropenem and tigecycline did not obtain the meropenem target, but did attain the 
tigecycline threshold. Although tigecycline was not administered until day 5 of therapy, we conservatively defined this patient as having achieved the requisite pharmacodynamic exposure.

Some limitations should be noted in this analysis. First, the number of patients included in the CARE Study was small and may not have been large enough to identify differences in exposure versus response. Second, we classified plazomicin, tigecycline, and meropenem pharmacodynamic exposure based on the baseline MIC for each individual drug. Therefore, no considerations for additive or synergistic interactions were made. It is possible that despite not achieving their individual drug pharmacodynamic thresholds, the addition of plazomicin to tigecycline or meropenem may lower the MIC or the pharmacodynamic threshold into an acceptable range for the aggressive dosing regimens to achieve target attainment. For instance, plazomicin has demonstrated in vitro synergy in combination with meropenem against some CRE; combinations with tigecycline typically show additivity/indifference $[28,29]$. However, the meropenem and tigecycline MIC when combined with plazomicin has yet to be described. While this consideration limits the ability to draw conclusions on plazomicin monotherapy, it remains suggestive that inclusion of plazomicin in the treatment regimen was essential for the observed efficacy. Finally, three included patients (one tigecycline and two meropenem) did not have original concentration data available for the pharmacokinetic analysis, and model fitting for one additional meropenem patient was not optimal. As a result, covariate-based models were applied to estimate pharmacokinetic parameter estimates for these four patients. This was done so as to include as many patients as possible among this small dataset. We do not believe having the actual pharmacokinetic parameter estimates for the two meropenem patients would have altered the conclusions given the high meropenem MICs of the baseline CRE. Notably, the estimated tigecycline patient was among one of the patients who did not achieve their pharmacodynamic exposure. This patient received a 100-mg loading dose followed by
$50 \mathrm{mg}$ q12 h (i.e., standard dosing) and was infected with a $K$. pneumoniae with a tigecycline MIC of $2 \mathrm{mg} / \mathrm{L}$. Obtaining optimal pharmacodynamic exposure at this MIC is challenging with standard tigecycline dosing regimens [21].

\section{CONCLUSIONS}

In summary, we observed pharmacodynamic exposures to be suboptimal for tigecycline and meropenem in $40 \%$ of patients who participated in the CARE Study, while plazomicin achieved its threshold in all 15 patients. Microbiological response and clinical efficacy were observed in $100 \%(6 / 6)$ and $83.3 \%(5 / 6)$ of patients with low threshold attainment by tigecycline and meropenem dosing regimens, respectively. Provided plazomicin achieved its requisite pharmacodynamic exposure, optimization of tigecycline and meropenem therapy was not required for the combination to achieve microbiological response and clinical efficacy against serious CRE infections in the CARE study.

\section{ACKNOWLEDGEMENTS}

Funding. This study was funded by Achaogen, Inc (South San Francisco, CA, USA). This project has been funded in whole or in part with federal funds from the Biomedical Advanced Research and Development Authority, Office of the Assistant Secretary for Preparedness and Response, Office of the Secretary, Department of Health and Human Services, under Contract no. HHSO100201000046C. All authors had full access to all of the data in this study and take complete responsibility for the integrity of the data and accuracy of the data analysis.

Authorship. All named authors meet the International Committee of Medical Journal Editors (ICMJE) criteria for authorship for this article, take responsibility for the integrity of the work as a whole, and have given their approval for this version to be published. 
Disclosures. At the time of the study Aryun Kim was an employee of Achaogen, Inc. Dr Kim is now an employee at BioMarin Pharmaceuticals. At the time of the study Daniel J. Cloutier was an employee of Achaogen, Inc. Dr Cloutier is now an employee at VIR Biotechnology. Joseph Kuti is a member of the Achaogen Speakers Bureau. David Nicolau is a member of the Achaogen Speakers Bureau.

Compliance with Ethics Guidelines. The study was reviewed and approved by the Hartford Hospital Institutional Review Board. Informed consent was originally obtained for participation in the CARE study, and additional consent for these analyses was waived because all data were available and collected for the purposes of the CARE Study. We acknowledge Kristie Kooken and Alex Smith from Achaogen for assistance with data management and Julie Seroogy and Raymond Diokno for bioanalytical support.

Open Access. This article is distributed under the terms of the Creative Commons Attribution-NonCommercial 4.0 International License (http://creativecommons.org/licenses/ by-nc/4.0/), which permits any noncommercial use, distribution, and reproduction in any medium, provided you give appropriate credit to the original author(s) and the source, provide a link to the Creative Commons license, and indicate if changes were made.

\section{REFERENCES}

1. Gupta N, Limbago BM, Patel JB, et al. Carbapenemresistant Enterobacteriaceae: epidemiology and prevention. Clin Infect Dis. 2011;53:60-7.

2. Guh AY, Bulens SN, Mu Y, et al. Epidemiology of carbapenem-resistant Enterobacteriaceae in 7 US communities, 2012-2013. JAMA. 2015;314:147987.

3. Rice LB, Carias LL, Hutton RA, et al. The KQ element, a complex genetic region conferring transferable resistance to carbapenems, aminoglycosides, and fluoroquinolones in Klebsiella pneumoniae. Antimicrob Agents Chemother. 2008;52:3427-9.
4. Almaghrabi R, Clancy CJ, Doi Y, et al. Carbapenemresistant Klebsiella pneumoniae strains exhibit diversity in aminoglycoside-modifying enzymes, which exert differing effects on plazomicin and other agents. Antimicrob Agents Chemother. 2014;58:4443-51.

5. Cox G, Ejim L, Stogios PJ, et al. Plazomicin retains antibiotic activity against most aminoglycoside modifying enzymes. ACS Infect Dis. 2018;4:980-7.

6. Armstrong ES, Miller GH. Combating evolution with intelligent design: the neoglycoside ACHN490. Curr Opin Microbiol. 2010;13:565-73.

7. Livermore DM, Mushtaq S, Warner M, et al. Activity of aminoglycosides, including ACHN-490, against carbapenem-resistant Enterobacteriaceae isolates. J Antimicrob Chemother. 2011;66:48-53.

8. McKinnell JA, Dwyer JP, Talbot GH, et al. Plazomicin for infections caused by carbapenem-resistant Enterbacteriaceae. N Engl J Med. 2019;380:8.

9. Ambrose PG, Bhavnani SM, Rubino CM, et al. Pharmacokinetics-pharmacodynamics of antimicrobial therapy: it's not just for mice anymore. Clin Infect Dis. 2007;44:79-86.

10. Trang M, Seroogy JD, Van Wart SA, et al. Population pharmacokinetic analyses for plazomicin using pooled data from phase 1, 2, and 3 clinical studies. Antimicrob Agents Chemother. 2019;63:e0232918.

11. Neely MN, van Guilder MG, Yamada WM, et al. Accurate detection of outliers and subpopulations with Pmetrics, a nonparametric and parametric pharmacometric modeling and simulation package for R. Ther Drug Monit. 2012;34:467-76.

12. Bhavnani SM, Rubino CM, Hammel JP, et al. Pharmacological and patient-specific response determinants in patients with hospital-acquired pneumonia treated with tigecycline. Antimicrob Agents Chemother. 2012;56:1065-72.

13. Crandon JL, Ariano RE, Zelenitsky SA, et al. Optimization of meropenem dosage in the critically ill population based on renal function. Intensive Care Med. 2011;37:632-8.

14. Grupper M, Kuti JL, Nicolau DP. Continuous and prolonged intravenous beta-lactam dosing: implications for the clinical laboratory. Clin Microbiol Rev. 2016;29:759-72.

15. Rubino CM, Forrest A, Bhavnani SM, et al. Tigecycline population pharmacokinetics in patients with community- or hospital-acquired pneumonia. Antimicrob Agents Chemother. 2010;54:5180-6. 
16. Bhavnani SM, Hammel JP, Trang M, et al. Pharmacokinetic-pharmacodynamic target attainment analyses to support plazomicin dose selection and recommendations for interpretive criteria for in vitro susceptibility testing for Enterobacteriaceae (poster no. 518). In: American Society for Microbiology Microbe 2018, Atlanta, GA; 2018.

17. Daikos GL, Jackson GG, Lolans VT, et al. Adaptive resistance to aminoglycoside antibiotics from firstexposure down-regulation. J Infect Dis. 1990;162: 414-20.

18. Daikos GL, Lolans VT, Jackson GG. First-exposure adaptive resistance to aminoglycoside antibiotics in vivo with meaning for optimal clinical use. Antimicrob Agents Chemother. 1991;35:117-23.

19. Karlowsky JA, Zelenitsky SA, Zhanel GG. Aminoglycoside adaptive resistance. Pharmacotherapy. 1997;17:549-55.

20. Abdelraouf $\mathrm{K}$, Kim $\mathrm{A}$, Krause $\mathrm{KM}$, et al. In vivo efficacy of plazomicin alone or in combination with meropenem or tigecycline against Enterobacteriaceae isolates exhibiting various resistance mechanisms in an immunocompetent murine septicemia model. Antimicrob Agents Chemother. 2018;62: e01074-18.

21. Xie J, Roberts JA, Alobaid AS, et al. Population pharmacokinetics of tigecycline in critically ill patients with severe infections. Antimicrob Agents Chemother. 2017;61:e00345-17.

22. Borsuk-De Moor A, Rypulak E, Potrec B, et al. Population pharmacokinetics of high-dose tigecycline in patients with sepsis or septic shock. Antimicrob Agents Chemother. 2018;62:e02273-17.
23. Korth-Bradley JM, Troy SM, Matschke K, et al. Tigecycline pharmacokinetics in subjects with various degrees of renal function. J Clin Pharmacol. 2012;52:1379-87.

24. Passarell JA, Meagher AK, Liolios K, et al. Exposureresponse analyses of tigecycline efficacy in patients with complicated intra-abdominal infections. Antimicrob Agents Chemother. 2008;52:204-10.

25. Koomanachai P, Kim A, Nicolau DP. Pharmacodynamic evaluation of tigecycline against Acinetobacter baumannii in a murine pneumonia model. J Antimicrob Chemother. 2009;63:982-7.

26. Li C, Du X, Kuti JL, et al. Clinical pharmacodynamics of meropenem in patients with lower respiratory tract infections. Antimicrob Agents Chemother. 2007;51:1725-30.

27. Crandon JL, Luyt CE, Aubry A, et al. Pharmacodynamics of carbapenems for the treatment of Pseudomonas aeruginosa ventilator-associated pneumonia: associations with clinical outcome and recurrence. J Antimicrob Chemother. 2016;71:2534-7.

28. Rodriguez-Avial I, Pena I, Picazo JJ, et al. In vitro activity of the next-generation aminoglycoside plazomicin alone and in combination with colistin, meropenem, fosfomycin or tigecycline against carbapenemase-producing Enterobacteriaceae strains. Int J Antimicrob Agents. 2015;46:616-21.

29. Thwaites M, Hall D, Stoneburner A, et al. Activity of plazomicin in combination with other antibiotics against multidrug-resistant Enterobactericeae. Diagn Microbiol Infect Dis. 2018;92:338-45. 